\title{
Construction of various soliton solutions via the simpli- fied extended sinh-Gordon equation expansion method
}

\author{
Tukur Abdulkadir Sulaiman ${ }^{1,2}$, Hasan Bulut ${ }^{1,3, *}$, and Haci Mehmet Baskonus ${ }^{4}$ \\ ${ }^{1}$ Department of Mathematics, Firat University, Elazig, Turkey \\ ${ }^{2}$ Department of Mathematics, Federal University, Dutse, Jigawa, Nigeria \\ ${ }^{3}$ Department of Mathematics Education, Final International University, Kyrenia, Cyprus \\ ${ }^{4}$ Department of Computer Engineering, Munzur University, Tunceli, Turkey
}

\begin{abstract}
In this paper, we present the simplified version of the extended sinh-Gordon equation expansion method. The newly proposed approach is based on the well-known sinh-Gordon equation and a travelling wave transformation. We successfully employed this approach to the $(2+1)$-dimensional nonlinear Chiral Schrodinger's and various solitary wave solutions to the studied nonlinear model are successfully constructed. The $(2+1)$-dimensional nonlinear Chiral Schrodinger's equation describes the edge states of the fractional quantum hall effect. The 2D and 3D surfaces of some of the obtained solutions are plotted.
\end{abstract}

\section{Introduction}

Nonlinear evolution equations (NEEs) and their solutions play a vita role in the field of nonlinear science. These equations describe various nonlinear physical aspects that arise in the field of nonlinear science such as fluid mechanics, plasma physics, optic fibers, solid state physics etc. Various studies have been conducted on such type of equations by different scientists from all over the world [1-9].

However, in this study, the extended sinh-Gordon equation expansion method (ShGEEM) is simplified, in the sense that all the ansatzs use for obtaining the values of the parameters involved are derived from one equation $\left(\theta^{\prime}=\sqrt{\sigma} \cosh (\theta)\right)$, which leads to using single system of algebra equations from where the values of the parameters involved can be obtained. To test the simplicity and efficiency of the proposed approach, we apply it in investigating the $(2+1)$-dimensional Chiral nonlinear Schrödinger equation [10].

The $(2+1)$-dimensional Chiral nonlinear Schrödinger equation is given by [10]

$$
i q_{t}+a\left(q_{x x}+q_{y y}\right)+i\left(b_{1}\left(q q_{x}^{*}-q^{*} q_{x}\right)+b_{2}\left(q q_{y}^{*}-q^{*} q_{y}\right)\right) q=0
$$

where $q$ is the complex function of $x$ and $t, a$ is the coefficient of the dispersion terms and $b_{1}, b_{2}$ are nonlinear coupling constants.

The $(2+1)$-dimensional Chiral nonlinear Schrödinger equation describes the edge states of the fractional quantum hall effect [10].

\footnotetext{
*Corresponding Author: hbulut@firat.edu.tr
} 


\section{The simplified extended ShGEEM}

In this section, we present the general facts of the proposed scheme.

To search for the new travelling wave solutions to a given NEE by using the proposed scheme, the following analysis is carried out:

Consider the following equation:

$$
F\left(q, q q_{x}, q^{2} q_{t}, q q_{t t}, \ldots\right)=0,
$$

where $q$ is a function of the independent variable $x, t$ and the subscripts indicate the derivative of $q$ with respect to the independent variables and $F$ is the polynomial of the function $q$.

Consider the following sinh-Gordon equation [11]:

$$
q_{x t}=\lambda \sinh (q)
$$

where $q$ is the unknown function of $x, t$ and $\lambda \in \mathbb{R} \backslash\{0\}$.

Using the following wave transformation

$$
q=\Psi(\xi), \quad \xi=x+\vartheta t
$$

on Eq. (3), we get the following nonlinear ordinary differential equation (NODE);

$$
\Psi^{\prime \prime}=\frac{\lambda}{\vartheta} \sinh (\Psi)
$$

where $\Psi=\Psi(\xi), \xi$ is the amplitude and $\vartheta$ the velocity of the travelling wave respectively. Integrating Eq. (5), yields

$$
\left[\left(\frac{\Psi}{2}\right)^{\prime}\right]^{2}=\frac{2 \lambda}{\vartheta} \sinh ^{2}\left(\frac{\Psi}{2}\right)+\frac{\lambda \delta}{\vartheta}
$$

where $\delta$ is the integration constant, resulting from integrating Eq. (5).

Setting $\theta=\frac{\Psi}{2}$ and $\sigma=\frac{2 \lambda}{\vartheta}=\frac{\lambda \delta}{\vartheta}$, yields

$$
\theta^{\prime}=\sqrt{\sigma} \cosh (\theta)
$$

Eq. (7) is a variables separable equation, simplifying it, yields the following significant solutions:

$$
\begin{aligned}
& \cosh (\theta)=\tan (\sqrt{\sigma}(\xi+d)) \text { or } \cosh (\theta)=\cot (\sqrt{\sigma}(\xi+d)), \\
& \sinh (\theta)=\sec (\sqrt{\sigma}(\xi+d)) \text { or } \sinh (\theta)=\csc (\sqrt{\sigma}(\xi+d)),
\end{aligned}
$$

where $d$ is the integration constant, resulting from integrating Eq. (7).

When $\sigma=-1$ and $d=0$, Eq. (8) and (9) become

$$
\cosh (\theta)=-i \tanh (\xi) \text { or } \cosh (\theta)=i \operatorname{coth}(\xi)
$$


and

$$
\sinh (\theta)=\operatorname{sech}(\xi)) \text { or } \sinh (\theta)=i \operatorname{csch}(\xi)
$$

respectively, where $i=\sqrt{-1}$.

To find the new solutions to Eq. (2), we consider the following equation:

$$
\Psi(\theta)=\sum_{k=1}^{m} \cosh ^{k-1}(\theta)\left[B_{k} \sinh (\theta)+A_{k} \cosh (\theta)\right]+A_{0},
$$

and the set of series solutions

$$
\begin{gathered}
\Psi(\xi)=\sum_{k=1}^{m} \tan ^{k-1}(\sqrt{\sigma}(\xi+d))\left[B_{k} \sec (\sqrt{\sigma}(\xi+d)) \pm A_{k} \tan (\sqrt{\sigma}(\xi+d))\right]+A_{0}, \\
\Psi(\xi)=\sum_{k=1}^{m} \cot ^{k-1}(\sqrt{\sigma}(\xi+d))\left[B_{k} \csc (\sqrt{\sigma}(\xi+d)) \pm A_{k} \cot (\sqrt{\sigma}(\xi+d))\right]+A_{0}, \\
\Psi(\xi)=\sum_{k=1}^{m} \tanh ^{k-1}(\xi)\left[B_{k} \operatorname{sech}(\xi) \pm i A_{k} \tanh (\xi)\right]+A_{0}, \\
\Psi(\xi)=\sum_{k=1}^{m} \operatorname{coth}^{k-1}(\xi)\left[i B_{k} \operatorname{csch}(\xi) \pm i A_{k} \operatorname{coth}(\xi)\right]+A_{0} .
\end{gathered}
$$

The value of $m$ is determined by balancing the highest power nonlinear term and the highest derivative in the reduced NODE. Setting each summation of the coefficients of $\theta^{\prime} \sinh ^{i}(\theta) \cosh ^{j}(\theta),(s=0,1,0 \leq k \leq m, 0 \leq j \leq m)$ with the same power to zero, produces a set of algebraic equations. Solving this set of algebraic equations with the aid of the Wolfram Mathematical package, yields the values of the coefficients involved. Inserting the obtained values of coefficients into one of Eq. (13), (14), (15) or (16) along with $m$, gives the new travelling wave solutions to Eq. (2).

\section{Application}

In this section, we present the application of the simplified extended ShGEEM to the $(2+1)$-dimensional Chiral nonlinear Schrödinger equation [10] given in Eq. (1).

Consider the following complex wave transformation:

$$
q=\Psi(\xi) e^{i \Omega}, \xi=\alpha x+\beta y-\vartheta t, \Omega=r x+s y+\omega t+\varphi
$$

into Eq. (1), we get the relation

$$
\vartheta=2 a(\alpha r+\beta s)
$$

from the imaginary part and

$$
a\left(\alpha^{2}+\beta^{2}\right) \Psi^{\prime \prime}+2\left(r b_{1}+s b_{2}\right) \Psi^{3}-\left(a\left(r^{2}+s^{2}\right)+\omega\right) \Psi=0
$$

from the real part. 
Balancing the terms $\Psi^{\prime \prime}$ and $\Psi^{3}$ in Eq. (19), gives $m=1$.

Thus, Eq. (12), (13), (14), (15) and (16) take the following forms

$$
\begin{gathered}
\Psi(\theta)=B_{1} \sinh (\theta)+A_{1} \cosh (\theta)+A_{0}, \\
\Psi(\xi)=B_{1} \sec (\sqrt{\sigma}(\xi+d)) \pm A_{1} \tan (\sqrt{\sigma}(\xi+d))+A_{0}, \\
\Psi(\xi)=B_{1} \csc (\sqrt{\sigma}(\xi+d)) \pm A_{1} \cot (\sqrt{\sigma}(\xi+d))+A_{0}, \\
\Psi(\xi)=B_{1} \operatorname{sech}(\xi) \pm i A_{1} \tanh (\xi)+A_{0}, \\
\Psi(\xi)=i B_{1} \operatorname{csch}(\xi) \pm i A_{1} \operatorname{coth}(\xi)+A_{0} .
\end{gathered}
$$

Substituting Eq. (20) and its second derivative into Eq. (19), we get an equation in hyperbolic functions. After making some hyperbolic function identities substitutions, we collect a group of algebraic equations by equating each summation of the coefficients of the hyperbolic functions with the same power to zero. To get the solutions to Eq. (1), we insert the obtained values of the coefficients into one of Eq. (21), (22), (23) and (24).

Case-I: $A_{0}=0, A_{1}=B_{1}=-\frac{\sqrt{-\sigma \omega\left(\alpha^{2}+\beta^{2}\right)}}{\sqrt{2\left(-2 s^{2}-2 r^{2}+\sigma\left(\alpha^{2}+\beta^{2}\right)\right)\left(r b_{1}+s b_{2}\right)}}$, $a=\frac{2 \omega}{\sigma\left(\alpha^{2}+\beta^{2}\right)-2 s^{2}-2 r^{2}}$.

Case-II: $A_{0}=0, A_{1}=B_{1}=-\frac{\sqrt{a^{3 / 2} \sigma\left(\alpha^{2}+\beta^{2}\right)\left(\sqrt{2\left(a\left(-2 s^{2}+\sigma\left(\alpha^{2}+\beta^{2}\right)\right)-2 \omega\right)} b_{1}+2 s b_{2} \sqrt{a}\right)}}{2 \sqrt{\left(a\left(-2 s^{2}+\sigma\left(\alpha^{2}+\beta^{2}\right)\right)-2 \omega\right) b_{1}^{2}-2 a s^{2} b_{2}^{2}}}$, $r=-\frac{\sqrt{a\left(-2 s^{2}+\sigma\left(\alpha^{2}+\beta^{2}\right)\right)-2 \omega}}{\sqrt{2 a}}$.

Utilizing these coefficients with Eq. (21), (22), (23) and (24)), we pursue the following set of solutions:

Set-1: The singular periodic wave solutions

Substituting the coefficients in case-I into Eq. (21) and (22), we have

$$
\begin{array}{r}
q_{1}(x, y, t)=\frac{\sqrt{-\sigma \omega\left(\alpha^{2}+\beta^{2}\right)}}{\sqrt{2\left(-2 s^{2}-2 r^{2}+\sigma\left(\alpha^{2}+\beta^{2}\right)\right)\left(r b_{1}+s b_{2}\right)}} \\
\times\left( \pm \tan \left[\sqrt{\sigma}\left(d+\alpha x+\beta y-\frac{4 \omega(\alpha r+\beta s)}{\sigma\left(\alpha^{2}+\beta^{2}\right)-2 s^{2}-2 r^{2}} t\right)\right]\right. \\
\left.-\sec \left[\sqrt{\sigma}\left(d+\alpha x+\beta y-\frac{4 \omega(\alpha r+\beta s)}{\sigma\left(\alpha^{2}+\beta^{2}\right)-2 s^{2}-2 r^{2}} t\right)\right]\right) e^{i(r x+s y+\omega t+\varphi)},
\end{array}
$$




$$
\begin{array}{r}
q_{2}(x, y, t)=-\frac{\sqrt{-\sigma \omega\left(\alpha^{2}+\beta^{2}\right)}}{\sqrt{2\left(-2 s^{2}-2 r^{2}+\sigma\left(\alpha^{2}+\beta^{2}\right)\right)\left(r b_{1}+s b_{2}\right)}} \\
\times \cot \left[\frac{1}{2} \sqrt{\sigma}\left(d+\alpha x+\beta y-\frac{4 \omega(\alpha r+\beta s)}{\sigma\left(\alpha^{2}+\beta^{2}\right)-2 s^{2}-2 r^{2}} t\right)\right] e^{i(r x+s y+\omega t+\varphi)}, \\
q_{3}(x, y, t)=-\frac{\sqrt{-\sigma \omega\left(\alpha^{2}+\beta^{2}\right)}}{\sqrt{2\left(-2 s^{2}-2 r^{2}+\sigma\left(\alpha^{2}+\beta^{2}\right)\right)\left(r b_{1}+s b_{2}\right)}} \\
\times \tan \left[\frac{1}{2} \sqrt{\sigma}\left(d+\alpha x+\beta y-\frac{4 \omega(\alpha r+\beta s)}{\sigma\left(\alpha^{2}+\beta^{2}\right)-2 s^{2}-2 r^{2}} t\right)\right] e^{i(r x+s y+\omega t+\varphi)},
\end{array}
$$

where $\sigma>0$ for valid solution.

Substituting the coefficients in case-II into Eq. (21) and (22), we have

$$
\begin{array}{r}
q_{4}(x, y, t)=\frac{\sqrt{a^{3 / 2} \sigma\left(\alpha^{2}+\beta^{2}\right)\left(\sqrt{2\left(a\left(-2 s^{2}+\sigma\left(\alpha^{2}+\beta^{2}\right)\right)-2 \omega\right)} b_{1}+2 s b_{2} \sqrt{a}\right)}}{2 \sqrt{\left(a\left(-2 s^{2}+\sigma\left(\alpha^{2}+\beta^{2}\right)\right)-2 \omega\right) b_{1}^{2}-2 a s^{2} b_{2}^{2}}} \\
\times\left( \pm \tan \left[\sqrt{\sigma}\left(d+\alpha x+\beta y-2 a\left(\beta s-\frac{\alpha \sqrt{a\left(\sigma\left(\alpha^{2}+\beta^{2}\right)-2 s^{2}\right)-2 \omega}}{\sqrt{2 a}}\right) t\right)\right]\right. \\
\left.-\sec \left[\sqrt{\sigma}\left(d+\alpha x+\beta y-2 a\left(\beta s-\frac{\alpha \sqrt{a\left(\sigma\left(\alpha^{2}+\beta^{2}\right)-2 s^{2}\right)-2 \omega}}{\sqrt{2 a}}\right) t\right)\right]\right) \\
\left.\times e^{i\left(s y-\frac{\sqrt{a\left(-2 s^{2}+\sigma\left(\alpha^{2}+\beta^{2}\right)\right)-2 \omega}}{\sqrt{2 a}} x+\omega t+\varphi\right.}\right),
\end{array}
$$

$$
\begin{array}{r}
q_{5}(x, y, t)=-\frac{\sqrt{a^{3 / 2} \sigma\left(\alpha^{2}+\beta^{2}\right)\left(\sqrt{2\left(a\left(-2 s^{2}+\sigma\left(\alpha^{2}+\beta^{2}\right)\right)-2 \omega\right)} b_{1}+2 s b_{2} \sqrt{a}\right)}}{2 \sqrt{\left(a\left(-2 s^{2}+\sigma\left(\alpha^{2}+\beta^{2}\right)\right)-2 \omega\right) b_{1}^{2}-2 a s^{2} b_{2}^{2}}} \\
\times \cot \left[\frac{1}{2} \sqrt{\sigma}\left(d+\alpha x+\beta y-2 a\left(\beta s-\frac{\alpha \sqrt{a\left(\sigma\left(\alpha^{2}+\beta^{2}\right)-2 s^{2}\right)-2 \omega}}{\sqrt{2 a}}\right) t\right)\right. \\
\times e^{i\left(s y-\frac{\sqrt{a\left(-2 s^{2}+\sigma\left(\alpha^{2}+\beta^{2}\right)\right)-2 \omega}}{\sqrt{2 a}} x+\omega t+\varphi\right)},
\end{array}
$$

$$
\begin{array}{r}
q_{6}(x, y, t)=-\frac{\sqrt{a^{3 / 2} \sigma\left(\alpha^{2}+\beta^{2}\right)\left(\sqrt{2\left(a\left(-2 s^{2}+\sigma\left(\alpha^{2}+\beta^{2}\right)\right)-2 \omega\right)} b_{1}+2 s b_{2} \sqrt{a}\right)}}{2 \sqrt{\left(a\left(-2 s^{2}+\sigma\left(\alpha^{2}+\beta^{2}\right)\right)-2 \omega\right) b_{1}^{2}-2 a s^{2} b_{2}^{2}}} \\
\times \tan \left[\frac{1}{2} \sqrt{\sigma}\left(d+\alpha x+\beta y-2 a\left(\beta s-\frac{\alpha \sqrt{a\left(\sigma\left(\alpha^{2}+\beta^{2}\right)-2 s^{2}\right)-2 \omega}}{\sqrt{2 a}}\right) t\right)\right. \\
\left.\times e^{i\left(s y-\frac{\sqrt{a\left(-2 s^{2}+\sigma\left(\alpha^{2}+\beta^{2}\right)\right)-2 \omega}}{\sqrt{2 a}} x+\omega t+\varphi\right.}\right),
\end{array}
$$


where $\sigma, 2 a\left(a\left(\sigma\left(\alpha^{2}+\beta^{2}\right)-2 s^{2}\right)-2 \omega\right)>0$ for valid solution.

Set-2: Combine dark-bright soliton solutions

Substituting the coefficients in case-I into Eq. (23), we have

$$
\begin{array}{r}
q_{7}(x, y, t)=\frac{\sqrt{\omega\left(\alpha^{2}+\beta^{2}\right)}}{\sqrt{2\left(2 s^{2}+2 r^{2}+\alpha^{2}+\beta^{2}\right)\left(r b_{1}+s b_{2}\right)}} \\
\times\left(i \operatorname{sech}\left[\alpha x+\beta y+\frac{4(\alpha r+\beta s)}{2 s^{2}+2 r^{2}+\alpha^{2}+\beta^{2}} t\right]\right. \\
\left. \pm \tanh \left[\alpha x+\beta y+\frac{4(\alpha r+\beta s)}{2 s^{2}+2 r^{2}+\alpha^{2}+\beta^{2}} t\right]\right) e^{i(r x+s y+\omega t+\varphi)},
\end{array}
$$

where $2 \omega\left(\alpha^{2}+\beta^{2}\right)\left(2 s^{2}+2 r^{2}+\alpha^{2}+\beta^{2}\right)\left(r b_{1}+s b_{2}\right)>0$ for valid soliton.

Substituting the coefficients in case-II into Eq. (23), we have

$$
\begin{array}{r}
q_{8}(x, y, t)=\frac{\sqrt{-a^{3 / 2}\left(\alpha^{2}+\beta^{2}\right)\left(\sqrt{-2 a\left(2 s^{2}+\alpha^{2}+\beta^{2}\right)-4 \omega} b_{1}+2 s b_{2} \sqrt{a}\right)}}{2 \sqrt{\left(a\left(2 s^{2}+\alpha^{2}+\beta^{2}\right)+2 \omega\right) b_{1}^{2}+2 a s^{2} b_{2}^{2}}} \\
\times\left(i \operatorname{sech}\left[\alpha x+\beta y-2 a\left(\beta s-\frac{\alpha \sqrt{-a\left(2 s^{2}+\alpha^{2}+\beta^{2}\right)-2 \omega}}{\sqrt{2 a}}\right) t\right]\right. \\
\left. \pm \tanh \left[\alpha x+\beta y-2 a\left(\beta s-\frac{\alpha \sqrt{-a\left(2 s^{2}+\alpha^{2}+\beta^{2}\right)-2 \omega}}{\sqrt{2 a}}\right) t\right]\right) \\
\left.\times e^{i\left(s y-\frac{\sqrt{-a\left(2 s^{2} \alpha^{2}+\beta^{2}\right)}}{\sqrt{2 a}} x+\omega t+\varphi\right.}\right),
\end{array}
$$

where $a^{3 / 2}\left(\alpha^{2}+\beta^{2}\right)<0,\left(2 a\left(2 s^{2}+\alpha^{2}+\beta^{2}\right)+4 \omega\right)<0,2 a\left(a\left(2 s^{2}+\alpha^{2}+\beta^{2}\right)+2 \omega\right)<0$, $\left(a\left(2 s^{2}+\alpha^{2}+\beta^{2}\right)+2 \omega\right) b_{1}^{2}+2 a s^{2} b_{2}^{2}>0$ and $a>0$ for valid soliton.

Set-3: The dark soliton solutions

Substituting the coefficients in case-I into Eq. (24), we have

$$
\begin{array}{r}
q_{9}(x, y, t)=\frac{\sqrt{\omega\left(\alpha^{2}+\beta^{2}\right)}}{\sqrt{2\left(2 s^{2}+2 r^{2}+\alpha^{2}+\beta^{2}\right)\left(r b_{1}+s b_{2}\right)}} \\
\times \tanh \left[\frac{1}{2}\left(\alpha x+\beta y+\frac{4 \omega(\alpha r+\beta s)}{2 s^{2}+2 r^{2}+\alpha^{2}+\beta^{2}} t\right)\right] e^{i(r x+s y+\omega t+\varphi)},
\end{array}
$$

where $2 \omega\left(\alpha^{2}+\beta^{2}\right)\left(2 s^{2}+2 r^{2}+\alpha^{2}+\beta^{2}\right)\left(r b_{1}+s b_{2}\right)>0$ for valid soliton.

Substituting the coefficients in case-II into Eq. (24), we have 


$$
\begin{array}{r}
q_{10}(x, y, t)=\frac{\sqrt{-a^{3 / 2}\left(\alpha^{2}+\beta^{2}\right)\left(\sqrt{-2 a\left(2 s^{2}+\alpha^{2}+\beta^{2}\right)-4 \omega} b_{1}+2 s b_{2} \sqrt{a}\right)}}{2 \sqrt{\left(a\left(2 s^{2}+\alpha^{2}+\beta^{2}\right)+2 \omega\right) b_{1}^{2}+2 a s^{2} b_{2}^{2}}} \\
\times \tanh \left[\frac{1}{2}\left(\alpha x+\beta y-2 a\left(\beta s-\frac{\alpha \sqrt{-a\left(2 s^{2}+\alpha^{2}+\beta^{2}\right)-2 \omega}}{\sqrt{2 a}}\right) t\right)\right] \\
\times e^{i\left(s y-\frac{\sqrt{-a\left(2 s^{2} \alpha^{2}+\beta^{2}\right)}}{\sqrt{2 a}} x+\omega t+\varphi\right)},
\end{array}
$$

where $a^{3 / 2}\left(\alpha^{2}+\beta^{2}\right)<0,\left(2 a\left(2 s^{2}+\alpha^{2}+\beta^{2}\right)+4 \omega\right)<0,2 a\left(a\left(2 s^{2}+\alpha^{2}+\beta^{2}\right)+2 \omega\right)<0$, $\left(a\left(2 s^{2}+\alpha^{2}+\beta^{2}\right)+2 \omega\right) b_{1}^{2}+2 a s^{2} b_{2}^{2}>0$ and $a>0$ for valid soliton.

Set-4: Singular soliton solutions

Substituting the coefficients in case-I into Eq. (24), we have

$$
\begin{array}{r}
q_{11}(x, y, t)=-\frac{\sqrt{\omega\left(\alpha^{2}+\beta^{2}\right)}}{\sqrt{2\left(2 s^{2}+2 r^{2}+\alpha^{2}+\beta^{2}\right)\left(r b_{1}+s b_{2}\right)}} \\
\times \operatorname{coth}\left[\frac{1}{2}\left(\alpha x+\beta y+\frac{4 \omega(\alpha r+\beta s)}{2 s^{2}+2 r^{2}+\alpha^{2}+\beta^{2}} t\right)\right] e^{i(r x+s y+\omega t+\varphi)} .
\end{array}
$$

Substituting the coefficients in case-I into Eq. (24), we have

$$
\begin{array}{r}
q_{12}(x, y, t)=-\frac{\sqrt{-a^{3 / 2}\left(\alpha^{2}+\beta^{2}\right)\left(\sqrt{-2 a\left(2 s^{2}+\alpha^{2}+\beta^{2}\right)-4 \omega} b_{1}+2 s b_{2} \sqrt{a}\right)}}{2 \sqrt{\left(a\left(2 s^{2}+\alpha^{2}+\beta^{2}\right)+2 \omega\right) b_{1}^{2}+2 a s^{2} b_{2}^{2}}} \\
\\
\times \operatorname{coth}\left[\frac{1}{2}\left(\alpha x+\beta y-2 a\left(\beta s-\frac{\alpha \sqrt{-a\left(2 s^{2}+\alpha^{2}+\beta^{2}\right)-2 \omega}}{\sqrt{2 a}}\right) t\right)\right] \\
\times e^{i\left(s y-\frac{\sqrt{-a\left(2 s^{2} \alpha^{2}+\beta^{2}\right)}}{\sqrt{2 a}} x+\omega t+\varphi\right)},
\end{array}
$$

where $2 a\left(a\left(2 s^{2}+\alpha^{2}+\beta^{2}\right)+2 \omega\right)<0$ for valid soliton.

\section{Results and Discussion}

In this section, we discuss the previously presented analytical techniques which have been developed by using the concept of the sinh-Gordon equation and tell the differences with our newly presented simplified approach. Under the choice of suitable values of the parameters, we also present the two- and three-dimensional plots to some of the obtained solutions obtained in this study.

Yan [12] proposed a sinh-Gordon equation expansion method which obtains the Jacobi elliptic function solutions to some nonlinear wave equations. In Yan [12], Eq. (3) is transformed into nonlinear ordinary differential equation (Eq. (5)) by using Eq. (4). When simplifying Eq. (5) by Yan [12], the constant of integration is considered to be nonzero and the following Jacobi elliptic functions are obtained: 


$$
\sinh (\theta)=\operatorname{cs}(\xi ; m) \text { and } \cosh (\theta)=n s(\xi ; m) .
$$

It is known that when $m \rightarrow 1, \operatorname{cs}(\xi ; m) \rightarrow \operatorname{csch}(\xi)$ and $n s(\xi ; m) \rightarrow \operatorname{coth}(\xi)$ and when $m \rightarrow 0, \operatorname{cs}(\xi ; m) \rightarrow \cot (\xi)$ and $n s(\xi ; m) \rightarrow \csc (\xi)$. For details, see Yan [12].

Xian-Lin and Jia-Shi [13] extended sinh-Gordon equation expansion method developed by Yan [12]. In the extended sinh-Gordon equation expansion method trigonometric and hyperbolic functions solutions can directly be secured. When simplifying Eq. (5), Xian-Lin and Jia-Shi [13] also considered the constant of integration to be zero in the following way:

In Eq. (6), Xian-Lin and Jia-Shi [13] considered $\frac{2 \lambda}{\vartheta}=a$ and $\frac{\lambda \delta}{\vartheta}=b$ so that the following equation is secured:

$$
\theta^{\prime}=\sqrt{a \sinh ^{2}(\theta)+b}
$$

Thus, Xian-Lin and Jia-Shi [13] solved Eq. (38) considering the following two cases:

Case-I: When $a=1, b=0$, Eq. (38) becomes

$$
\theta^{\prime}=\sinh (\theta)
$$

Case-II: When $b=1, a=1$, Eq. (38) becomes

$$
\theta^{\prime}=\cosh (\theta) \text {. }
$$

Case-I is solved to give hyperbolic functions solutions and Case-II is solved to give trigonometric functions solutions. This leads to having two individual polynomial equations in the power of hyperbolic functions $\left(\theta^{\prime} \sinh ^{k}(\theta) \cosh ^{j}(\theta)\right),(s=$ $0,1$ and $k, j=0,1,2, \ldots)$ as $\theta^{\prime}$ varies for each case. For details, see Xian-Lin and Jia-Shi [13].

In this study, we transform the same sinh-Gordon equation Eq. (3) as in Yan [12], Xian-Lin and Jia-Shi [13] by using Eq. (4). We also consider a nonzero constant of integration when simplifying Eq. (5), but in different way with Yan [12], Xian-Lin and Jia-Shi [13], as it can be seen from Eq. (6) in section 2. Doing this, we obtain Eq. (7). Eq. (7) is a variable separable equation, we simplify it and obtain the following trigonometric function solutions:

$$
\begin{aligned}
& \cosh (\theta)=\tan (\sqrt{\sigma}(\xi+d)) \text { or } \cosh (\theta)=\cot (\sqrt{\sigma}(\xi+d)), \\
& \sinh (\theta)=\sec (\sqrt{\sigma}(\xi+d)) \text { or } \sinh (\theta)=\csc (\sqrt{\sigma}(\xi+d)) .
\end{aligned}
$$

When we consider $\sigma=-1$ and $d=0$ in Eq. (41) and (42), we directly obtain the following hyperbolic function solutions:

$$
\begin{gathered}
\cosh (\theta)=-i \tanh (\xi) \text { or } \cosh (\theta)=i \operatorname{coth}(\xi), \\
\sinh (\theta)=\operatorname{sech}(\xi)) \text { or } \sinh (\theta)=i \operatorname{csch}(\xi) .
\end{gathered}
$$

Putting these solutions together, we have the forms of solutions to Eq. (2) presented in section 2 (that is Eq. (13), (14), (15) and (16)) which can be used to obtain different travelling wave solutions to various nonlinear evolution equations. 
It can be seen that in this study, all the solutions can be generated from Eq. (7) $(\sqrt{\sigma} \cosh (\theta))$ which overcome the use of two individual polynomial equations in the power of hyperbolic functions $\left(\theta^{\prime} s \sinh ^{k}(\theta) \cosh ^{j}(\theta)\right),(s=0,1$ and $k, j=0,1,2, \ldots)$ as we have only one $\theta^{\prime}$. Thus, one single polynomial equation can be used in collecting the system of algebraic equations and this is enough to obtained the solutions of the considered nonlinear model after obtaining the values of the parameters involved by solving the system of algebraic equations.

\section{Conclusion}

In this study, we present the simplified version of the extended sinh-Gordon equation expansion method. The efficiency of the simplified approach is tested on the $(2+1)$-dimensional nonlinear Chiral Schrödinger equation. We successfully construct singular periodic wave, dark soliton, combine dark-bright soliton, singular soliton and combine singular soliton solutions to these nonlinear complex models. We observed that the simplified sinh-Gordon equation expansion method is easy, direct and powerful mathematical approach that can be used to solved various nonlinear evolution equations.

\section{References}

[1] H. Bulut, H.A. Isik and T.A. Sulaiman, ITM Web of Conferences 13, 01019 (2017)

[2] X.J. Yang, J.A.T. Machado and D. Baleanu, Fractals 25(4), 1740006 (2017)

[3] A. Neirameh, Fractals, 55, 2839-2847 (2016)

[4] S. Bibi and S.T. Mohyud-Din, Journal of the Association of Arab Universities for Basic and Applied Sciences 15 90-93 (2014)

[5] T.A. Sulaiman, T. Akturk, H. Bulut and H.M. Baskonus, Journal of Electromagnetic Waves and Applications 32(9), 1093-1105 (2017)

[6] S. Duran, M. Askin and T.A. Sulaiman, IJOCTA 7(3), 240-247 (2017)

[7] A. Yokus, H.M. Baskonus, T.A. Sulaiman and H. Bulut, Numer Methods Partial Differential Eq. 34, 211-227 (2018)

[8] A.M. Wazwaz and S.A. El-Tantawy, Nonlinear Dynamics 83(3) 1529-1534 (2016)

[9] R.I. Nuruddeena, L. Muhammad, A.M. Nass and T.A. Sulaiman, Palestine Journal of Mathematics 7(1) 262-280 (2018)

[10] H. Bulut, T.A. Sulaiman and B. Demirdag, Nonlinear Dyn. 91 1985-1991 (2018)

[11] M.A. Ablowitz and P.A. Clarkson, Solitons, Nonlinear Evolution Equations and Inverse Scattering, (University Press, Cambridge, (1991)) 115-235

[12] Z. Yan, MMRC, AMSS, Academia Sinica, Beijing 22 363-375 (2003)

[13] X. Xian-Lin and T. Jia-Shi, Commun. Theor. Phys. 50, 1047 (2008) 